\title{
Reflection on and Exploration of a Standardized Resident Training Programme Based on a Comprehensive Examination of Clinical Skills
}

\author{
Jing Yang ${ }^{1, \#}$, Chun-ting Lu ${ }^{2, \#}$, Dan Wang ${ }^{2, \#}$, Bi-yao $\mathrm{Su}^{2} \&$ Si-min Huang ${ }^{1}$ \\ ${ }^{1}$ Department of Internal Medicine, The First Affiliated Hospital, Jinan University, Guangzhou, China \\ ${ }^{2}$ The Science \& Education Office, The First Affiliated Hospital, Jinan University, Guangzhou, China \\ \#: These authors have contributed equally to the work. \\ Correspondence: Si-min Huang, Department of Internal Medicine, The First Affiliated Hospital, Jinan University, \\ Guangzhou, 510630, China.
}

Received: February 22, 2018

Accepted: March 14, 2018

Online Published: April 3, 2018

doi:10.5430/irhe.v3n2p17

URL: https://doi.org/10.5430/irhe.v3n2p17

\begin{abstract}
To identify the optimal clinical skills training programme for internal medicine residents by reflecting upon and analysing the usual training plan and arrangement, and practical tests according to the national standardized resident training policies and requirements. The 3-year-rotation and training plan of the 2013 and 2014 internal medicine resident cohorts, the basic skills and case analysis scores on the basic entrance examination and final graduation examination were reflected, summarized and compared with the ultimate purpose of evaluating standardized resident training. This knowledge was used to perfect a competency-oriented training system that integrated clinical theory, clinical scenarios, clinical skills, bedside teaching, learning, etc. After a 3-year programme of standardized training, the residents' clinical skills were significantly improved. Statistically significant enhancements $(P<0.05)$ were observed in the case analysis and clinical skills test scores between the basic entrance and graduation examinations of the trainees in both the 2013 and 2014 cohorts. No significant differences $(P>0.05)$ were observed between the trainees in the 2013 and 2014 cohorts in their graduation scores on the clinical skills and case analysis tests, suggesting that both cohorts achieved the same level of mastery and reached the standardized training target, which also reflected the homogeneity of the residency training programme. While this study indicated that the training method was effective, exploring better models in the future is warranted. In conclusion, as a national policy, continuously incorporating reflection and exploration is necessary for designing a reasonable programme for standardized resident training. In this study, analysis of the basic entrance and graduation examinations of comprehensive clinical skills were important for developing an effective standardized resident training programme and training team with satisfactory teaching effects. By this way, implementing and starting new training methods gradually will be improved and especially carried out from 2015 cohorts. Therefore, the residents' clinical competence will be enhanced and also for undergraduates' and postgraduates' education as well. At last, the national education criteria for residency will be met.
\end{abstract}

Keywords: standardized resident training, clinical skills, basic entrance examination, final graduation examination

\section{Introduction}

The first guidelines for national standardized training bases for residents were issued by the National Health and Family Planning Commission of the People's Republic of China in October 2014. Our college, the First Clinical College of Jinan University, was the first resident training base. A standardized residency training system is an important national policy. Training systems should be both problem-oriented and demand-oriented, and competence cultivation must be a core requirement in comprehensive medical education systems for post-graduation residents. The education system should consider the students' continuing medical education. Therefore, by comprehensively upgrading at the specialized technical level, the professionalism of all medical personnel in the health care and family planning field can be achieved(Wang \& Chen, 2017). International residency training goals are also consulted, such as the medical educational system in the United States. The Accreditation Council for Graduate Medical Education (ACGME) has issued 6 core competencies for all physicians: i) medical knowledge; ii) patient care; iii) communication; iv) professionalism; v) practice-based learning; vi) systems-based practice (details on the ACGME web site, www.acgme.org)(Charap, Levin, Pearlman, \& Blaser, 2005; Crain et al., 2005). 
As a teaching hospital, our college has undertaken teaching tasks at all levels, including undergraduate, postgraduate, resident and specialties. Residency training is an important teaching task. In addition, our training and teaching curriculum is continually improving due to the development and implementation of a standardized training system for residents. A pilot study was initiated in 2013, and a fully standardized training programme has been implemented since 2015. By reflecting upon and exploring the basic entrance and final graduation examinations of clinical skills, the effectiveness of a standardized resident programme can be perfected(S. Y. Zhang, Pan, et al., 2015). This study reflected on the training mode of residents admitted in 2013 (2013 cohort) and 2014 (2014 cohort) and its efficacy. It also explored the contours of an optimal clinical skills training program for internal medicine. These objectives were achieved by analysing and comparing the average scores on the basic entrance examination and final graduation examination, which assessed case analysis skills, clinical thinking skills, and operation techniques. In addition, the residents' clinical skills training efficiency and mastery was assessed by initial and terminal evaluation. Throughout this process, a scientific and reasonable residency training and evaluation system was gradually established. This system can be applied in the standardized training for residents enrolled in 2015 (2015 cohort)(S. Y. Zhang, Ye, et al., 2015). The construction of the teacher's team is also beneficial to all clinical practice teaching including undergraduate, postgraduate, resident and specialties.

\section{Method}

\subsection{Participants and Setting}

Trainees who were enrolled at our college in 2013 and 2014 and participated in the standardized resident training programme for the internal medicine major were included. All participants had completed their undergraduate medical education and were undergoing 3 years of standardized training.

\subsection{Study Method Objectives}

2.2.1 To reflect upon and summarize the 3-year-rotation and training plan of the 2013 and 2014 internal medicine resident cohorts.

2.2.2 To compare the scores on the basic entrance examinations with those on the final graduation examinations with respect to case analysis and basic skills in the 2013 and 2014 cohorts to evaluate the standardized training effects.

2.2.3 To analyse the characteristics of and differences between the 2013 and 2014 cohorts prior to the actual internal medicine training programme and at graduation using their scores on the clinical skills tests.

2.2.4 To explore and implement a new competency-oriented training system integrating clinical theory, clinical scenarios, clinical skills, bedside teaching, learning, etc.

2.2.5 To establish a training team that includes teaching management members, teaching faculty, and specialists who are responsible for rotation arrangement, clinical training, evaluation, etc. To review the requirements and objectives of the national standardized resident training policy and refine and analyse the assessment process and evaluation criteria to implement new training programmes for the 2015 cohort.

\subsection{Statistic Analysis}

The analysis was performed using SPSS version 16.0. The quantitative data are expressed as median values and inter-quartile ranges (IQRs). The comparisons between the groups were performed using the Mann-Whitney U test for data with a non-parametric distribution. A $P$-value of 0.05 or less was considered statistically significant.

\section{Results}

\subsection{Reflection on and Summation of the Standardized Resident Training Rotation Plan and Evaluation of the Programme Efficacy in the 2013 and 2014 Cohorts}

According to the national standardized training policy and rules (details in http://www.gdzpgl.net/), all internal medicine residents should complete a 3-year-rotation (illustrated in Table 1) of training in compulsory departments, including cardiovascular disease (including electrocardiogram, ECG), respiratory, gastroenterology, urinary, haematology, endocrine and metabolism, rheumatology and immunology, infectious diseases, neurology, emergency medicine, out-patient clinic and critical care units. Optional rotations in other departments include medical imaging, ultrasonic medicine, nuclear medicine, geriatric medicine, dermatology, oncology, community health care, etc. 
Table 1. Arrangement and rotation of internal medicine resident

\begin{tabular}{|c|c|c|}
\hline & Rotation department & Duration(months) \\
\hline \multirow{13}{*}{ Compulsory } & Cardiovascular disease(including electrocardiogram, ECG) & 4 \\
\hline & Respiratory & 3 \\
\hline & Gastroenterology & 3 \\
\hline & Urinary & 2 \\
\hline & Haematology & 2 \\
\hline & Endocrine and metabolism & 2 \\
\hline & Infectious diseases & 2 \\
\hline & Rheumatology and immunology & 2 \\
\hline & Neurological medicine & 2 \\
\hline & Emergency medicine & 3 \\
\hline & Out-patient clinic & 2 \\
\hline & Critical care units & 2 \\
\hline & Total & 29 \\
\hline \multirow{6}{*}{ Optional } & Medical imaging, ultrasonic medicine, nuclear medicine & \\
\hline & Geriatric medicine & \\
\hline & Dermatology & \\
\hline & Oncology & \\
\hline & Community health care & \\
\hline & Total & 4 \\
\hline
\end{tabular}

In our country, according to the new policy "Standardized Resident Training Objectives and Requirements", the residents at our hospital undergo a comprehensive assessment of professional ethics, clinical knowledge, clinical skills, etc. All trainees must pass the rotation evaluations, including the usual competency manifestations and theoretical and practical tests in each department throughout the training process. Before graduating, the trainees must also complete the final comprehensive examinations, including public courses, clinical theory, clinical practice, etc. Among these examinations, the clinical practical examinations are administered by the internal medicine department to independently assess the residents' clinical competencies and abilities related to common diseases and urgent problems. An analysis of the final examinations could be helpful in establishing and improving the standardized training programme for residency.

3.2 A comparison of the scores between the basic entrance and final graduation examinations with respect to case analysis and basic skills of the 2013 and 2014 cohorts is shown in Table 2.

Table 2. Comparison the scores between the basic entrance examination and final graduation examinations with respect to case analysis and basic skills tests of the 2013 and 2014 cohorts

\begin{tabular}{|c|c|c|c|c|}
\hline \multirow[b]{2}{*}{ Examination items } & \multicolumn{2}{|c|}{ Grade 2013} & \multicolumn{2}{|c|}{ Grade 2014} \\
\hline & $\begin{array}{l}\text { Case } \\
\text { analysis }^{\#}\end{array}$ & $\begin{array}{l}\text { Clinical } \\
\text { skills }^{\#}\end{array}$ & $\begin{array}{l}\text { Case } \\
\text { analysis }\end{array}$ & Clinical basic skills \\
\hline Basic entrance examination scores & $65(50-80)$ & $70(67-77)$ & $81(70-83.7)$ & $70(60.5-80)$ \\
\hline Graduation examination scores & $84(81.5-89)$ & $88.8(85-90.3)$ & $85(81-89.3)$ & $88.8(85-91.9)$ \\
\hline $\mathrm{Z}$ & 4.91 & 5.25 & 3.75 & 5.49 \\
\hline$P$ & $0.000 * *$ & $0.000 * *$ & $0.000 * *$ & $0.000 * *$ \\
\hline
\end{tabular}

\footnotetext{
\#: Data were expressed as median values and inter-quartile ranges (IQRs).
}

**: $P<0.01$. 
3.3 A comparison of the performance and difference between the 2013 and 2014 cohorts on the case analysis and basic skills tests during the basic entrance and graduation examinations is shown in Table 3.

Table 3. Comparing the performance and difference between grade 2013 and grade 2014 on case analysis and basic skills tests during the basic entrance and graduation examination

\begin{tabular}{llcccc}
\hline \multicolumn{2}{c}{ Examination items } & Grade 2013 & Grade 2014 & Z & $P$ \\
\hline \multirow{2}{*}{ Basic entrance examination } & Case analysis $^{\#}$ & $65(50-80)$ & $81(70-83.7)$ & 2.15 & $0.031^{*}$ \\
& Clinical basic skills $^{*}$ & $70(67-77)$ & $70(60.5-80)$ & 0.50 & 0.61 \\
\multirow{2}{*}{ Graduation examination } & Case analysis $^{*}$ & $84(81.5-89)$ & $85(81-89.3)$ & 0.07 & 0.94 \\
& Clinical basic skills\# $^{\#}$ & $88.8(85-90.3)$ & $88.8(85-91.9)$ & 0.13 & 0.90 \\
\hline
\end{tabular}

\#: Data were expressed as median values and inter-quartile ranges (IQRs).

$*: P<0.05$.

3.4 By exploring the entrance and graduation examinations, the training plan, clinical teaching scenarios and assessment standards were evaluated. Table 4 illustrates the checklist and evaluation items used on the entrance and final examinations.

3.4.1 Arrangement and Checklists of Clinical Skills Tests showed in Table 4.

Table 4. Arrangement and checklists of clinical skills tests

\begin{tabular}{|c|c|c|c|}
\hline Stations & Main Items of Evaluation & $\begin{array}{l}\text { Standard } \\
\text { Scores }\end{array}$ & $\begin{array}{c}\text { Total } \\
\text { Scores }\end{array}$ \\
\hline \multirow{12}{*}{$\begin{array}{l}\text { Station } 1 \\
\text { History } \\
\text { Taking }\end{array}$} & The following main symptoms related to history taking: & & \multirow{12}{*}{100} \\
\hline & fever, oedema, loss of body weight, cough and expectoration, dyspnoea, palpitation, cyanosis, chest pain, & & \\
\hline & $\begin{array}{l}\text { abdominal pain, diarrhoea, haematemesis and haematochezia, jaundice, headache, vertigo, syncope, tic and } \\
\text { disturbance of consciousness, mucocutaneous haemorrhage, frequent micturition, urgent micturition, odynur } \\
\text { anuria, polyuria, haematuria, etc. }\end{array}$ & $\begin{array}{l}\text { onvulsion, } \\
\text { oliguria, }\end{array}$ & \\
\hline & 1. Doctor-patient communication methods and skills & 20 & \\
\hline & $\begin{array}{l}\text { 2. Inquiring about general information (name, gender, age, occupation, marriage, nationality, birth place, } \\
\text { admission date, recording date, connect and phone, sources, etc.) }\end{array}$ & 5 & \\
\hline & 3. Inquire about and summarize the chief complaints & 20 & \\
\hline & 4. Inquire about present illness history using 5 marks for each part as follows: & & \\
\hline & $\begin{array}{l}\text { (1) onset; (2) characteristics of the main symptoms or signs; (3) aetiology or precipitating factors; (4) } \\
\text { development and progression of illness; (5) associated symptoms; (6) diagnosis and treatment processes } \\
\text { prior to admission; and (7) general conditions (appetite, sleep, urination, bowel movement, change of body } \\
\text { weight, etc.) }\end{array}$ & 35 & \\
\hline & $\begin{array}{l}\text { 5. Prior medical history (previous health state, previous diseases, trauma and operations, allergic history to } \\
\text { food and drugs, preventive inoculation, blood transfusion history, etc.) }\end{array}$ & 5 & \\
\hline & 6. Personal and social history & 5 & \\
\hline & 7. Menstrual, marital and childbearing history & 5 & \\
\hline & 8. Family history & 5 & \\
\hline
\end{tabular}

The following items based on the case:

(1) vital signs and general appearance; (2) skin mucous membrane and lymph nodes; (3) eyes; (4) ears, nose, and mouth;

Station 2 (5) neck; (6) inspection and palpation of the chest and lung; (7) lung field percussion and lung auscultation; (8)

Physical inspection, palpation, and auscultation of the heart; (9) inspection and percussion of the heart; (10) peripheral vessel

Examination examination; (11) abdominal palpation; (12) inspection, auscultation and percussion of the abdomen; (13) cranial nerves

(PE) I, II, III, IV, V, VI, and VII; (14) cranial nerves V, VII, VIII, IX, X, XI, and XII; (15) motor nerve; (16) sensory nerve; and (17) Physiologic reflexes, pathological reflexes, etc.

1. Attitude and manner towards the examinee or SP and consciousness of patient-friendliness and dedication 10

2. Complete contents of PE 
$\begin{array}{lr}\text { 3. Correct sequence of PE } & 20\end{array}$

$\begin{array}{lr}\text { 4. Skilfulness during PE } & 20\end{array}$

$\begin{array}{lr}\text { 5. Answering relevant questions } & 20\end{array}$

The following four punctures: thoracentesis, abdominocentesis, bone marrow aspiration, and lumber puncture

$\begin{array}{ll}\text { 1. Preparatory work } & 20\end{array}$

Station 3 2. Determining patient position and puncture site 10

Internal 3. Disinfecting the skin; opening and checking the package and instruments; placing surgical drape; and 20

Medicine administering local anaesthesia

$\begin{array}{lll}\text { Punctures } & \text { 4. Needle penetration and specimen collection } & 10 \\ & \text { 5. Post-procedures } & 10\end{array}$

6. Concept and procedures related to disinfection; observing patient illness throughout the process 20

7. Answering relevant questions

10

Common diseases related to respiratory, cardiovascular, digestive, urinary, endocrine and metabolism, haematological, rheumatic and immune system disorders

1. Oral summary of the main clinical features for further diagnosis based on the case provided 40

Station 4 2. Proposing an hypothesis and making a primary diagnosis 5

Case 3. Describing the diagnostic clues 5

Analysis 4. Making a differential diagnosis 5

5. Analysing the laboratory investigations and assistant and instrumental examinations $\quad 15$

$\begin{array}{ll}\text { 6. Establishing a management plan } & 20\end{array}$

$\begin{array}{ll}\text { 7. Doctor-patient communication and humanistic care } & 20\end{array}$

Abbreviations: PE, physical examination; SP, standardized patient.

\subsubsection{An Example of a Clinical Scenario on the Final Examination of Case Analysis Prior to Graduation}

A 73-year-old male worker presented with a 10-year history of recurrent chest discomfort, palpitations, shortness of breath, and cough with a small amount of sputum accompanied by oedema of the bilateral lower extremities. The symptoms were aggravated for two hours, and he was then admitted to the hospital. Initially, these symptoms were only present after moderate physical activities, but the patient gradually developed dyspnoea even at rest and could not lie flat. Two hours prior to admission, the patient felt extremely uncomfortable and exhibited excessive sweating, chest tightness, and difficulty breathing. His prior medical history showed a 20 -year history of hypertension, and his blood pressure (BP) fluctuated in the range of 140 150/90 95 mmHg; his BP was controlled by anti-hypertensive drugs.

Upon the PE at admission, the following were noted: BP 195/115 mmHg; well-oriented; forceful sitting position and orthopnoea; and tachypnoea with excessive sweating. No jugular venous engorgement was observed. Wheezing and moist rales were heard in the base of both lungs. The cardiac border was enlarged to the left and downward. The heart rate (HR) was $150 \mathrm{bpm}$ with an irregular rhythm. The S1 volume was variable. A systolic murmur of III/6 degree was heard in the apex. Mild pitting oedema was observed in the lower extremities.

The assistant's investigations showed the following: ECG: ST-T change; Echocardiography: a serious enlargement of the left atria and left ventricle; an obvious weakness in the anterior wall contraction in the left ventricle; and a moderate to large mitral regurgitation. For this case, initially, the trainees must take a brief history and perform a focused physical examination within 20 minutes. Then, they should prescribe the appropriate tests and consider the possible diagnosis and further management based on the information provided. Therefore, the clinical skills and clinical thinking of the trainee are assessed during this stage. In addition, we could also design a specific clinical scenario that emphasizes the competency of invasive skills used in internal medicine punctures. This scenario could provide an assessment of the learners' clinical abilities, such as aseptic techniques, procedural knowledge, patient care, and urgent decision making.

\subsection{The Establishment of a Training Manager and Educator Team Has Been Proposed}

This team could include teachers, secretaries, mentors and experts responsible for the arrangement, instruction, evaluation and final examinations for multi-source assessment and feedback (MSAF)(Antonioni, 1996) throughout the process. A multi-level training management and implement team was composed as follows:

3.5.1 The teaching secretaries were responsible for implementing and arranging the residency rotations, entry education, regular training courses and entrance examinations. In addition, the secretaries recorded the scores in the 
national standardized resident training system. The teaching secretaries played an important role in guaranteeing that the residency training smoothly accommodated the needs of the diverse trainees and the challenging specialties in the internal medicine department. This supportive role also facilitated the transfer to the next department, which occurred after the trainees passed their entrance evaluation.

3.5.2 The supervisor and mentor teams were responsible for teaching and guiding the residents' clinical, training and scientific work. This team cultivated the skills that enhanced the residents' clinical comprehensive abilities, such as making diagnoses, deciding on treatments for common diseases and fostering the techniques of history taking, physical examinations, practical operations, case analyses, doctor-patient communications, humanistic care concepts, etc.

3.5.3 The team of internal medicine experts was responsible for collecting and studying the clinical cases on the entrance and final examinations. This team was also responsible for analysing the evaluation items, checklists and debriefing.

3.6 Based on a review of the national standardized resident training policy and an analysis of the teaching experience obtained during the training of the 2013 and 2014 cohorts, better didactic approaches were established that could be used to train the novice trainees in the 2015 cohort. A closer integration of the clinical scenarios and bedside teaching could be implemented within the framework of the usual medical work, rotation practical tests and initial and final examinations. In addition, the study could take advantage of the teaching hospital resources and clinical skills comprehensive centre platform by incorporating SP, simulations and high fidelity techniques to educate and enhance the residents' clinical competences and medical humanities quality. Furthermore, the combination of simulation training and clinical practice has greatly remedied the shortcomings of merely teaching theory without providing students with the opportunity to practise on real patients (Back et al., 2014; Yang, Huang, Li, Feng, \& Lu, 2018). This combination could improve the core clinical practice competencies of residents in various dimensions(Kirk, 2007; McGaghie, Issenberg, Barsuk, \& Wayne, 2014).

\section{Discussion}

4.1 After a 3-year standardized residency training programme, the residents' clinical skills were significantly improved. The training method was effective, but further improvement is needed and can be achieved by exploring better models in the future.

4.1.1 A statistically significant enhancement $(P<0.05)$ in case analysis and clinical skills test scores was observed between the basic entrance and graduation examinations in both the 2013 and 2014 cohorts. The progression from the entrance to graduation examination was observed in this programme. Thus, the effectiveness of the standardized residency training programme was satisfactory.

4.1.2 No significant differences $(P>0.05)$ were observed between the 2013 and 2014 cohorts in the graduation scores on the clinical skills and case analysis tests. Thus, both cohorts achieved the same degree of mastery and reached the standardized training target, which also reflects the homogeneity of the residency training system. It was also indicated that the standardized residency training needed to be consistent with the national policies and training goals, stability of management system and faculty team, scientific and reasonable training programs.

4.1.3 No significant difference was observed between the 2013 and 2014 cohorts on the basic entrance examination scores on the clinical skills tests. Thus, the clinical skill level of the enrolled and selected trainees was basically the same. In addition, the standardization of the evaluation criteria for the clinical skills test was more easily controlled and determined.

4.1.4 However, the scores on the case analysis component of the basic entrance examinations in the 2014 cohort were higher than those in the 2013 cohort. In addition, the difference between the cohorts was statistically significant $(P<0.05)$. This difference may have been caused by the difficulties in the standardized case analyses encountered in the practical test portion, which included scenarios that were influenced by complicated patient clinical information and materials related to their diagnosis and treatment. Therefore, unifying and exploring evaluation criteria for case analysis tests in the future are necessary. In addition, expertise in clinical thinking and comprehensive competence is gained by performing medical work in routine rotations, which can impact the design and implementation of case analysis examinations that more effectively mimic real clinical situations. Furthermore, the basic entrance and final graduation examinations could facilitate the construction of the competency-oriented standardized resident training evaluation system(B. Zhang \& Wang, 2015). 
4.1.5 During training, cultivating comprehensive clinical competency has always been considered an essential component according to the requirements and objectives of the standardized residency training national policy. Therefore, this study focused on training in medical ethics, policy regulations, clinical practice skills, professional theory knowledge, doctor-patient communication skills, etc. The key elements of the programme were to improve residents' clinical diagnosis and treatment skills, determine appropriate clinical teaching and foster scientific research activity. The most important purposes included enhancing the trainees' clinical skills to master professional knowledge, acquire clinical practice, and diagnose and treat common diseases under the guidance of a supervisor, while gradually mastering those clinical diagnoses and treatment pathways and understanding reasonable selections and applications of commonly used medicines and drugs. These achievements all help lay a solid basis for becoming a good doctor in the future. In addition, the recognition of situations that require rapid management and cardiopulmonary resuscitation for critically ill conditions is important.

4.1.6 Based on the national standardized resident training policy, requirements and objectives, the administration and training expert team which consisted of managers, topic expert and specialists had studied and established training syllabus, kinds of common diseases, number of cases and clinical skills, and clinical scientific researches for resdidents to master during the rotation in each section of internal medicine department.

4.2 In the usual and routine training programme, the basic combination of specialty instruction and a centralized and unified training mode were used. The residents' duties included the following: bedside management of patients, admission of new patients, medical rounds, day and night shift work, diagnosis and treatment of patients, rescue efforts for seriously ill patients, medical documentation and records, etc. These medical activities all help improve the residents' clinical competence. In the interim, the residents were required to participate in the following specific training: theoretical courses, clinical skills training courses, teaching rounds, case discussions and other academic activities. Accordingly, the formative and final evaluations were extremely important and helpful in assessing the training effects. The analysis and reflection in this study can help further improve the practice of standardized resident training.

4.3 In view of the problems, the training of basic clinical skills should be strengthened using simulation and clinical rotations and the teaching methods should be adapted to trainees' individual features(Brydges et al., 2017).

4.3.1 Many deficiencies and weaknesses were identified in the trainees' basic clinical skills training and tests, such as physical examination issues and internal punctures. These limitations can be summarized as follows: i) there was not enough humanistic care; ii) doctor-patient communication was not performed skilfully; iii) the physical examination performance lacked a standardized and correct approach. In the clinical practice teaching, the students who examined the patient were relatively blunt or rude in their attitude and manner. For example, in an abdominal examination, the learners tended to palpate using strong force that was not timed with the patient's abdominal respiration. Even in certain extremities, the trainees removed the patient's clothes without sufficient communication and explanation to the patients during the physical examination. These acts can cause patient dissatisfaction or complaints. In addition, other nonstandard operating problems, such as the lack of zero calibration at the beginning of a blood pressure measurement, no relaxation of the examining areas in lymph node palpation, wrong lung percussion methods, missing items in heart auscultation, a stiff technique of liver and spleen palpation, strong pressure on the thyroid glands and a lack of familiarity with neurological physical examinations, etc. were identified; and iv) during the internal punctures examinations, violations of aseptic rules and incorrect procedures were observed. Several learners usually confused the aseptic area and contaminated areas during the operation and did not seriously perform a preoperative assessment of the patients' illness. Other common mistakes that were harmful to the patients were observed in the practical tests as follows: not verifying the patient's name and or obtaining the informed consent signature, choosing the wrong position for the puncture, using incorrect disinfection concepts and methods, using the wrong procedures in operations and not observing the patient's situation before, during and after the process. Accordingly, the following guidance and instructions should be emphasized throughout the standardized training program. The patient's safety should be considered to reduce mistakes during each step of an operation. Learners should review the patient's illness and information, assess their preparatory work-ups and carefully review the puncture procedures each time. Aseptic operations must be considered and carefully performed. The residents must be trained in medical simulation centres until they are skilful. Using a comprehensive medical simulator, the residents were able to learn and practise acute clinical events without endangering the patient. Through this training, the residents were also able to address critical patients in a safe and controlled environment that strengthened their knowledge and improved their ability to solve problems. In addition, this programme could increase team work, cooperation and communication abilities(Lu, Huang, Li, Feng, \& Yang, 2017). Therefore, the trainees were repeatedly reminded to strengthen their skills through more practice and improvements in the future training 
programme. Clinical medicine is a highly practical discipline, and young doctors must master clinical skills and increase their clinical competence by availing themselves to many practical training opportunities that encompass many clinical situations. Only in this way, can standard and accurate clinical diagnoses, treatments and relevant techniques be improved and achieved.

4.3.2 Clinical thinking training requires a long period of education and promotion.

In the 2014 cohort, the case analysis tests on the graduation examination began with a history inquiry that introduced the clinical problems and common symptoms of that case. Several residents needed to strengthen their history-taking skills because they did not perform this task systematically and reasonably. Trainees took a history from a standardized patient (SP) based on the scenario designed by the teachers and then performed a focused and significant physical examination after accumulating the important information. Subsequently, the residents could make a primary diagnosis and establish a management plan for further evaluation, which is similar to a real clinical situation. This exploration and reform was closer to the specific clinical scenario and more vividly reflected the clinical situation. In addition, the evaluation of the teaching efficacy for the 2014 cohort was more standardized and objective than that for the 2013 cohort, which could overcome the uncertainty and difficulty in the determination of case analysis examination questions simultaneously. Thus, standardized resident training should be comprehensively performed in a multi-level programme that includes usual clinical practical work, teaching ward rounds, significant case discussions, specific lectures, practical skills and clinical thinking training. The flexible integration and combination of problem-based learning, case-based learning, and team-based learning could be used to enhance the clinical competency of residents. The teaching rounds' competition held by our college in 2017 were also a good attempt to standardize the specification of teaching round process and enhance residents' clinical practical competencies and team work abilities.

\section{Conclusion}

The analysis of the basic entrance and final graduation examinations of comprehensive clinical skills was important for reflection on and the exploration of a standardized resident training programme and teaching effects. This analysis was also helpful for the implementation and reform of new training methods to improve the residents' clinical competence step-by-step. In addition, the national education criteria for residency were met.

\section{Limitations and Future Directions}

In the future, clinical competence-oriented programmes and arrangements can enhance the effectiveness of standardized resident training. Starting with the 2015 cohort, we aim to increase the teaching cases, scenario collections and applications in the formative and final assessments. More teaching and learning methods are required and will be explored in resident training(Xie, Cao, \& Zhao, 2015).

\section{Acknowledgements}

The authors would like to thank the academic staff and residents who contributed to the study and the members of the usual teaching programme at the first clinical medicine college and the medical college of Jinan University. This study was financially supported by the Guangdong Medical Science and Technology Research Foundation (Grant No. 2018557), Teaching Research Programs of Higher Medical Education of Ministry of Education in 2016 (grant number: Edu-CR-2016-4-2, Edu-CR-2016-4-3 and Edu-CR-2016-6-2) and Education Teaching Reform Project of Guangdong Province (grant number: 85514022).

\section{Author contributions}

YJ, LCT and HSM performed this study and were involved in the conception, design, analysis, and interpretation of the results. WD and SBY participated in the design of the study, evaluated the medical records and collected and analysed the data. All authors read and approved the final manuscript.

\section{Compliance with ethical standards:}

Declaration of interest: The authors have no conflicts of interest to report.

Ethics and consent: The study was conducted in accordance with the Helsinki Declaration of 1975 as revised in 2008. The medical student participants were informed in written and oral remarks. Throughout the process, the patients' privacy was considered and protected, and no invasive or harmful procedures were performed on any patients. In addition, the study data were accessible to the authors.

\section{References}

Antonioni, D. (1996). Designing an effective 360-degree appraisal feedback process. Organizational Dynamics, 
25(2), 24-38. https://doi.org/10.1016/S0090-2616(96)90023-6

Back, D. A., Haberstroh, N., Antolic, A., Sostmann, K., Schmidmaier, G., \& Hoff, E. (2014). Blended learning approach improves teaching in a problem-based learning environment in orthopedics - a pilot study. BMC Med Educ, 14, 17. https://doi.org/10.1186/1472-6920-14-17

Brydges, R., Stroud, L., Wong, B. M., Holmboe, E. S., Imrie, K., \& Hatala, R. (2017). Core Competencies or a Competent Core? A Scoping Review and Realist Synthesis of Invasive Bedside Procedural Skills Training in Internal Medicine. Acad Med, 92(11), 1632-1643. https://doi.org/10.1097/ACM.0000000000001726

Charap, M. H., Levin, R. I., Pearlman, R. E., \& Blaser, M. J. (2005). Internal medicine residency training in the 21st century: aligning requirements with professional needs. Am J Med, 118(9), 1042-1046. https://doi.org/10.1016/j.amjmed.2005.06.009

Crain, B. J., Alston, S. R., Bruch, L. A., Hamilton, R. L., Mclendon, R. E., Rhldes, C. H., ... Weidenheim, K. M. (2005). Accreditation Council for GraduateMedical Education (ACGME) Competencies in Neuropathology Training. Journal of Neuropathology \& Experimental Neurology, 64(4), 273-279. https://doi.org/10.1093/jnen/64.4.273

Kirk, L. M. (2007). Professionalism in medicine: definitions and considerations for teaching. Proc (Bayl Univ Med Cent), 20(1), 13-16. https://doi.org/10.1080/08998280.2007.11928225

Lu, C. T., Huang, S. M., Li, Z. J., Feng, L., \& Yang, J. (2017). Effects of Exploratory and Heuristic Multi-methods on System-oriented Curricula Based on Clinical Scenarios. International Journal of Higher Education, 6(6), 55-65. https://doi.org/10.5430/ijhe.v6n6p55

McGaghie, W. C., Issenberg, S. B., Barsuk, J. H., \& Wayne, D. B. (2014). A critical review of simulation-based mastery learning with translationaloutcomes. Medical Education, 48, 375-385. https://doi.org/10.1111/medu.12391

Wang, Q. C., \& Chen, J. W. (2017). Evaluation of Standardized Residents Training. Hosp Admin J Chin PLA, 24(3), 276-277.

Xie, L. Y., Cao, G., \& Zhao, Y. (2015). Teaching Method Combining PBL with CBL in Standardized Training of Residents. Chinese Medical Record, 16(10), 74-76.

Yang, J., Huang, S. M., Li, Z. J., Feng, L., \& Lu, C. T. (2018). Integrating Simulation Scenarios and Clinical Practices Guided by Concepts of Translational Medicine. International Journal of Higher Education, 7(1), 10-11. https://doi.org/10.5430/ijhe.v7n1p10

Zhang, B., \& Wang, Z. Y. (2015). Construction of the Competency-oriented Standardized Residency Training Evaluation System. Chinese Hospital Management, 35(9), 48-50.

Zhang, S. Y., Pan, H., Xue, H. D., Zhu, H. J., Fang, W. G., \& Zhao, Y. P. (2015). Pactice and insights on standardized residency training at PUMCH. Chin J Hosp Admin, 31(12), 914-916.

Zhang, S. Y., Ye, W., Zheng, W. Y., Liu, S. C., Shao, C., H., P., \& Li, H. (2015). Initial study of stratifying management with objective structured clinical examination in resident standardized training. Basic \& Clinical Medicine, 35(9), 1299-1302. 